\title{
Síndrome de rubéola congénita en 6 colegios para niños con sordera y/o ceguera, en Lima, Cusco y Arequipa, Perú, 1998 - 2000
}

\author{
DORA BLITCHTEIN-WINICKI ${ }^{1}$, NAZARIO SILVA², MARIO NARVÁEZ², JORGE GONZÁLES ${ }^{3}$, \\ MARIBEL GONZÁLES ${ }^{1}$, ROBERTO DEL AGUILA ${ }^{1}$, LUIS SUAREZ-OGNIO ${ }^{1}$ y col. \\ ${ }^{1}$ Oficina General de Epidemiología OGE, Ministerio de Salud Perú. \\ ${ }^{2}$ Hospital Nacional Materno Infantil San Bartolomé. ${ }^{3}$ Instituto Nacional de Salud (INS).
}

\begin{abstract}
RESUMEN
OBJETIVO: Deteminar la presencia de casos de síndrome de rubéola congénita (SRC) en tres ciudades del Perú. DISEÑO: Transversal, descriptivo. MATERIAL Y MÉTODOS: En 6 colegios de educación especial para niños sordos y/o ciegos, ubicados en Lima (tres), Cusco (dos) y Arequipa (uno), se analizó la información contenida en registros médicos de niños menores de 12 años. Se incluyó los casos con criterios mayores de la definición de SRC. RESULTADOS: De 364 registros de niños que estudian en colegios especiales, se incluyó 105; de ellos, 64,7\% (75) tuvo manifestaciones del sistema nervioso, $31 \%$ oculares. Utilizando una ficha validada para el estudio, $61,9 \%$ correspondió a casos sospechosos, $21 \%$ probables y dos fueron casos confirmados $(1,9 \%)$. CONCLUSIONES: Debido a que 6,6\% (24) del total de niños que estudian en colegios especiales son casos probables y confirmados de SRC, se evidencia que éste es un problema que existe en el país, por lo cual se fundamenta la necesidad de iniciar la vacunación antirubéolica en el país.
\end{abstract}

Palabras clave: Síndrome de rubéola congénita; vacuna contra la rubéola; sordera; ceguera; colegio.

\section{CONGENITAL RUBELLA SYNDROME IN 6 SCHOOLS FOR DEAF AND/OR BLIND CHILDREN IN LIMA, CUSCO AND AREQUIPA, PERU, 1998-2000 SUMMARY}

OBJECTIVE: To determine the presence of congenital rubella syndrome (CRS) in three Peruvian cities. DESIGN: Transversal, descriptive study. MATERIAL AND METHODS: Children under twelve years old with major definition criteriae of congenital rubella syndrome receiving special education in Lima (three), Cusco (two) and Arequipa (one) schools, from 1998 to 2000, were included. RESULTS: From 364 children 105 were included, $61 \%$ of cases suspicious, $21 \%$ probable and $1,9 \%$ confirmed; $64,7 \%$ had central nervous system manifestations, $31 \%$ ocular manifestations, $18 \%$ at least two clinical manifestations. CONCLUSIONS: Probable and confirmed cases of CRS represented $6,6 \%$ of all children receiving special education, evidencing that CRS represents a health problem in our country and the need to initiate anti-rubella vaccination in Peru.

Key words: Rubella syndrome, congenital; rubella vaccine; deafness; blindness; School.

\footnotetext{
Correspondencia:

Dra. Dora Blitchtein-Winicki

Camilo Carrillo 402

Lima 11, Perú

E-mail: investigacion@oge.sld.pe dblit@terra.com.pe
} 


\section{INTRODUCCIÓN}

La rubéola es una enfermedad causada por Rubivirus, que se manifiesta con exantema moderado, genera baja morbilidad y pocas complicaciones en niños y adultos. Sin embargo, desde 1942, Norman Gregg describió que la exposición de gestantes a esta enfermedad durante el primer trimestre de embarazo causa defectos congénitos severos $\left({ }^{1}\right)$.

La pandemia mundial de rubéola de 1962 a 1965 puso al descubierto la importancia del síndrome de rubéola congénita (SRC). Se estima que solamente en Estados Unidos hubo más de 2000 casos de SRC y en 1964 afectó a 1\% de los embarazos. Desde 1969, el uso de vacunas con virus atenuados ha prevenido epidemias de rubéola. Los estudios de seroprevalencia para dicha enfermedad han demostrado que las mujeres, en muchos países en desarrollo, tienen baja seroinmunidad, particularmente en zonas rurales e islas. La rubéola es una infección exclusiva de los seres humanos y su único reservorio está representado por los casos de SRC, pudiendo excretar el virus durante meses $\left({ }^{2,3}\right)$.

Puede ocurrir en todos los continentes y poblaciones, independientemente de raza, sexo y nivel socioeconómico. Es un problema de salud pública, debido a los efectos teratogénicos en el feto durante el embarazo, que provoca abortos espontáneos y el nacimiento de niños con SRC. Estos niños presentan las manifestaciones históricamente descritas, tales como, catarata, malformaciones cardíacas, retraso mental y sordera; además, manifestaciones transitorias, como trombocitopenia, púrpura, hepatitis, neumonitis $\left.{ }^{4}\right)$, lesiones óseas, meningoencefalitis, aparición tardía de diabetes mellitus y panencefalitis progresiva $\left({ }^{5}\right)$.

El Sistema Regional de Vigilancia de Sarampión puso al descubierto a la rubéola como un problema de salud en Latinoamérica. Del total de los casos sospechosos de sarampión investigados por los laboratorios, en 1996, $17 \%$ tuvo como diagnóstico final rubéola. Los países que presentaron las frecuencias más elevadas de ca- sos de rubéola por medio de esta vigilancia fueron Nicaragua (38\%), El Salvador (33\%), Costa Rica (32\%) y Perú (20\%). En nuestro país, en 1998, se reportó al sistema de notificación de sarampión MESS un total de 824 casos confirmados de rubéola; de éstos, 424 (51\%) correspondió al sexo femenino y, de ellas, 72 $(17 \%)$ tenía entre 15 y 49 años de edad $\left(^{6}\right)$. Ante esta realidad, la Oficina General de Epidemiología (OGE), a partir de mayo de 1999, consideró necesario incluir a la rubéola como un daño sujeto a notificación obligatoria. Cabe mencionar que, en el Perú, la vacuna triple viral (Measles, Rubella, Mumps) no se encuentra dentro del esquema del Programa Ampliado de Inmunizaciones (PAI) $\left(^{7}\right)$.

La rubéola es endémica en la mayor parte de los países. La tasa de SRC en la época anterior a la vacunación (MMR) era 1 de cada 1000 nacidos vivos. En ausencia de epidemias, más de 20000 niños nacen con SRC al año, en las Américas $\left({ }^{3}\right)$.

Se ha evaluado la contribución del SRC a la sordera, comparando las tendencias temporales de esta última con aquellas de rubéola adquirida (Australia). Pocos países tienen datos suficientemente completos para realizar dichas comparaciones. Otros estudios han utilizado datos clínicos o históricos para diagnosticar sordera relacionada con rubéola, o han comparado la prevalencia de anticuerpos IgG específicos para rubéola entre niños con sordera y niños sanos. Se ha conducido investigaciones poblacionales, las cuales son muy complejas, debido a su alto costo y a la prevalencia relativamente baja de la sordera neurosensorial severa ( 1 a 3 por 1000 nacidos vivos). Por esta dificultad, muchas investigaciones han estimado la proporción de defectos, tales como ceguera o sordera causados por SRC, en vez de la tasa por 1000 nacidos vivos $\left({ }^{8}\right)$.

El Perú se cuenta entre los países con tasas de susceptibilidad mayores de 10 a $25 \%$ en mujeres en edad reproductiva (1967) $\left({ }^{3}\right)$. Un modelo matemático estimó la incidencia de SRC para las áreas rurales del Perú en 464 por 100,000 nacidos vivos $\left({ }^{9}\right)$; no obstante, a pesar 
de ser el SRC un daño sujeto a notificación $\left({ }^{10}\right)$, no se le registra o notifica. Por esta razón, no se cuenta con información sobre el número de casos de SRC al año. Existe una urgente necesidad de realizar investigaciones al respecto, puesto que el SRC es causa principal prevenible de defectos congénitos, discapacidad auditiva y ceguera, en los países en desarrollo.

El objetivo general del presente estudio fue obtener evidencia sobre el SRC en tres ciudades del país, identificando casos confirmados y probables del mismo en colegios especiales para ciegos y sordos.

\section{MATERIAL Y MÉTODOS}

En el año 2001, se llevó a cabo un estudio transversal y descriptivo. La información fue obtenida en forma retrospectiva, para lo cual se utilizó datos de registros médicos de niños menores de 12 años (edad hasta la cual reciben educación en colegios especiales) que asistieron entre enero de 1998 a junio del 2000 a colegios especiales.

Se seleccionó a centros educativos especiales que cumplieran con los siguientes criterios:

1. La respuesta de las 34 Direcciones de Salud enviada a la Oficina General de Epidemiología, acerca del número de colegios para niños con sordera y/o ceguera que hubieran estado en actividad en el periodo de enero de 1998 hasta junio de 2000 .

2. Que los colegios de educación especial contaran con registros médicos con información para el llenado de la ficha.

Los casos fueron clasificados como sospechosos, probables y confirmados, de acuerdo con la definición de centro de enfermedades comunicables (CDC), debido a que son datos provenientes de fuentes secundarias $\left({ }^{11}\right)$ :

Caso sospechoso de síndrome de rubéola congénita.- Un niño(a) que, de acuerdo a su registro médico, tuviera hallazgos clínicos com- patibles (por ejemplo: sordera neurosensorial o catarata congénita, sin evidencia de otra causa probable de este signo).

Caso probable de síndrome de rubéola congénita.- Un niño(a) que, de acuerdo a su registro médico, tuviera dos criterios mayores (por ejemplo: cataratas, glaucoma congénito, retinopatía pigmentaria, enfermedad cardíaca congénita, sordera neurosensorial) o un criterio mayor y uno menor (por ejemplo: hepatomegalia, microcefalia, retraso en el crecimiento intrauterino, peso bajo al nacer, trombocitopenia, ictericia, enfermedad ósea radiolúcida) sin confirmación por el laboratorio. Si los criterios comprometieran al mismo órgano (por ejemplo: catarata y glaucoma), se tomaba en cuenta como uno solo.

Caso confirmado de síndrome de rubéola congénita.- Todo niño(a) de 6 a 12 meses de edad que tuviera serología positiva para rubéola (IgM ó 2 IgG positivos, tomados con un mes de diferencia) y signos consistentes con el diagnóstico del síndrome de rubéola congénita (SRC).

Se excluyó los casos que presentaron un diagnóstico clínico que por sí mismo explicara la presencia de signos compatibles con el síndrome de rubéola congénita (por ejemplo: síndromes genéticos, sífilis congénita, infección por citomegalovirus, etc.).

Se utilizó una ficha de datos, la cual fue validada con fines del estudio: validez del contenido, criterio de 7 expertos y un piloto (revisión de 32 registros médicos), en una escuela para niños sordos.

Para el análisis de los datos, la información fue ingresada en el software EpiInfo V.6,1 (CDC, Atlanta), utilizando una base de datos diseñada para el estudio (SRC2001), y se evaluó con este mismo paquete estadístico. Se realizó un análisis descriptivo de frecuencias.

Seis establecimientos educativos cumplieron con los criterios de selección para el estudio; de ellos, tres se encuentran localizados en Lima, dos en Cusco y uno en Arequipa. 


\section{RESULTADOS}

De un total de 364 niños que estudiaban en colegios especiales, 105 niños $(28 \%)$ cumplieron con tener cuando menos uno de estos signos: catarata o glaucoma congénitos, retinitis pigmentosa, sordera neurosensorial o malformación cardiaca congénita, de acuerdo con sus registros médicos, y $6,6 \%(24 / 364)$ de los niños fue caso probable y confirmado de síndrome de rubéola congénita (Tabla 1 y Figura).

De los 105 niños incluidos, dos $(1,9 \%)$ fueron casos confirmados, 22 (21\%) probables, 65 $(61,9 \%)$ sospechosos, mientras que $16(15,2 \%)$ tuvieron otros diagnósticos (Tabla 2).

Del total de criterios mayores en los 105 niños incluidos, $75(71,4 \%)$ presentó manifestaciones del sistema nervioso, $36(34,2 \%)$ afecciones oculares y $5(4,7 \%)$ compromiso cardiovascular (Tabla 3).
Entre las características demográficas de la población seleccionada (105) con criterios mayores, $80 \%$ (84) de los registros médicos perteneció a niños entre 3 y 11 años y $63,8 \%$ al sexo femenino $(n=67)($ Tabla 4$) ; 21,9 \%$ de estos niños (23) tuvo como lugar de nacimiento su domicilio.

En 2 registros médicos de casos, se confirmó el diagnóstico de síndrome de rubéola congénita, con pruebas de IgM para rubéola. Los casos citados fueron niñas de 2 y 6 años de edad, registrados en el Centro Educativo Nuestra Señora del Carmen, en Cusco.

Con respecto a sus antecedentes, en ambos casos la madre desarrolló una erupción cutánea (rash) durante el primer trimestre del embarazo. Como criterios de inclusión, las dos pacientes presentaron sordera neurológica y retraso mental. En una de ellas se evidenció catarata. En cuanto a los criterios menores, una niña tuvo

Tabla 1.- Registros médicos con criterios de inclusión por colegios especiales y departamento. Perú, enero 1998 - junio 2000.

\begin{tabular}{|c|c|c|c|c|c|}
\hline \multirow[t]{2}{*}{$\begin{array}{l}\text { Departa- } \\
\text { mento }\end{array}$} & \multirow[t]{2}{*}{ Establecimiento } & \multirow[t]{2}{*}{$\begin{aligned} & \text { Niños } \\
\geq & 12 \text { años }\end{aligned}$} & \multirow{2}{*}{$\begin{array}{c}\text { Niños con } \\
\text { un criterio } \\
\text { mayor } *\end{array}$} & \multicolumn{2}{|c|}{$\begin{array}{c}\text { Casos probables y } \\
\text { confirmados de SRC }\end{array}$} \\
\hline & & & & $\mathrm{n}$ & $\%$ \\
\hline \multirow[t]{3}{*}{ Lima } & Centro Peruano de Audición, Lenguaje y Aprendizaje & 117 & 56 & 14 & 12,0 \\
\hline & Centro Educativo San Francisco de Asís ${ }^{\dagger}$ & 98 & 10 & 3 & 3,1 \\
\hline & Hospital Hipólito Unanue ${ }^{\dagger \ddagger}$ & 32 & 10 & - & 0,0 \\
\hline Arequipa & Centro Educativo Especial Nuestra Señora del Pilar ${ }^{\dagger}$ & 49 & 13 & 3 & 6,1 \\
\hline \multirow[t]{2}{*}{ Cusco } & Centro Educativo Nuestra Señora del Carmen ${ }^{\dagger \ddagger}$ & 38 & 11 & 2 & 5,3 \\
\hline & Centro Educativo San Francisco de Asís ${ }^{\ddagger}$ & 30 & 5 & 2 & 6,7 \\
\hline Total & & 364 & 105 & 24 & 6,6 \\
\hline
\end{tabular}

\footnotetext{
* Criterio mayor: catarata o glaucoma congénitos, retinitis pigmentosa, sordera neurosensorial y/o malformación cardíaca congénita.

$\dagger$ Niños con ceguera.

* Niños con sordera.
} 


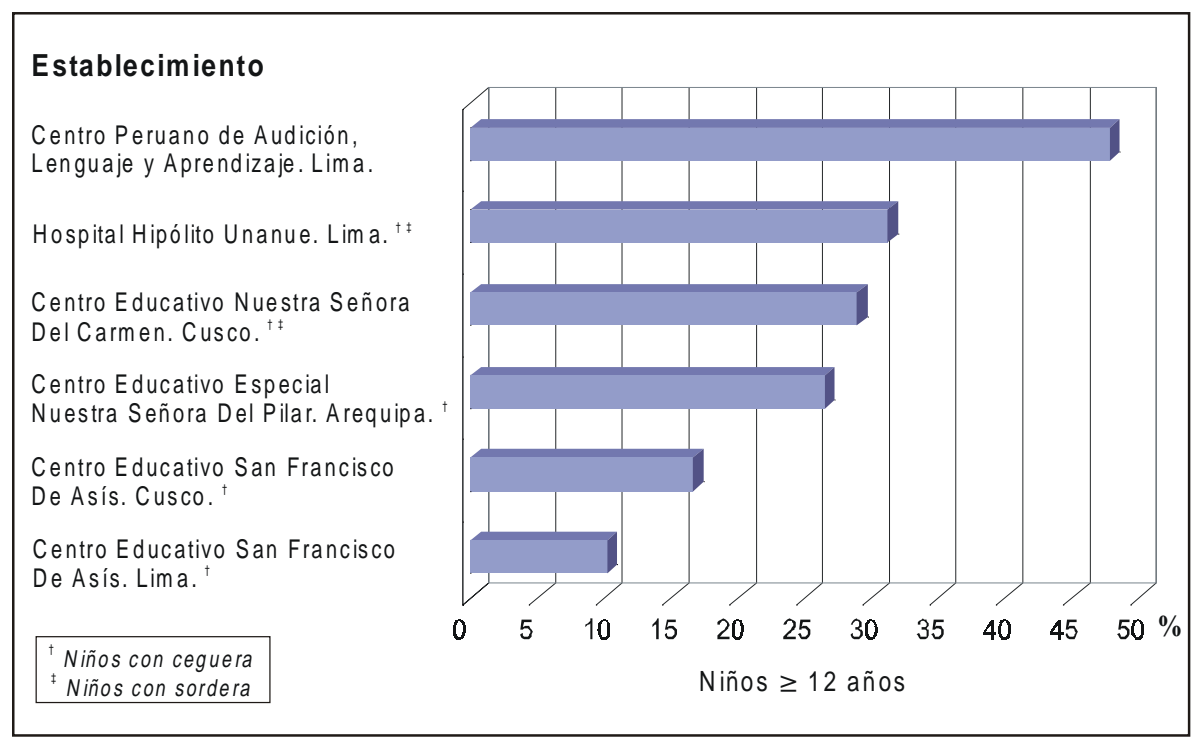

Figura.- Porcentaje de niños con al menos un criterio mayor (catarata o glaucoma congénito, retinitis pigmentosa, sordera neurosensorial o malformación cardíaca congénita).

Perú 1998 - 2000.

Tabla 2.- Clasificación de caso de síndrome de rubéola congénita.

\begin{tabular}{lrr}
\hline & $\mathrm{n}$ & \multicolumn{1}{c}{$\%$} \\
\hline Casos sospechosos & 65 & 61,9 \\
Casos probables & 22 & 21,0 \\
Casos confirmados & 2 & 1,9 \\
Otros diagnósticos & 16 & 15,2 \\
Total & 105 & 100,0 \\
\hline
\end{tabular}

peso bajo al nacer y la otra presentó neumonitis (Tabla 5).

\section{DISCUSIÓN}

La presencia de 24 casos probables y confirmados (22 y 2 , respectivamente) de SRC representa $23 \%$ del total de niños incluidos en el estudio y $6,6 \%$ del total de la población de niños que estudiaban. Con ello se confirmó la exis- tencia del síndrome de rubéola congénita como un problema de salud pública en el país. Los resultados del presente estudio (23\% de 105 niños entre 2 a 12 años) coinciden con otros sobre prevalencia de SRC realizados en colegios para niños sordos en India $\left({ }^{12}\right)$ y Malasia $\left({ }^{8}\right)(29 \%$ de

Tabla 3.- Criterios mayores de SRC.

\begin{tabular}{lrr}
\hline & $\mathrm{n}$ & $\%$ \\
\hline Manifestaciones oculares & 36 & 34,3 \\
Retinitis & 3 & 2,9 \\
Glaucoma & 9 & 8,6 \\
Catarata congénita & 24 & 22,9 \\
Manifestaciones cardiovasculares & 5 & 4,8 \\
Persistencia de conducto arterioso & 1 & 0,9 \\
Comunicación interauricular & 4 & 3,8 \\
Manifestaciones neurológicas & 75 & 71,4 \\
Meningoencefalitis & 3 & 2,9 \\
Retraso mental & 7 & 6,7 \\
Sordera neurogénica & 65 & 61,9 \\
& & \\
Total & 105 & 100,0
\end{tabular}


Tabla 4.- Sexo de los niños con síndrome de rubéola congénita

\begin{tabular}{lcc}
\hline Sexo & $\mathrm{n}$ & $\%$ \\
\hline Masculino & 38 & 36,2 \\
Femenino & 67 & 63,8 \\
Total & 105 & 100,0 \\
\hline
\end{tabular}

374 niños entre 3-14 años y 35\% de 165 niños de 7-14 años, respectivamente). En el Reino Unido, se estima que el SRC fue responsable de $15 \%$ de los casos de sordera neurosensorial y det $2 \%$ de las malformaciones cardiacas congénitas en niños, para la época anterior a la vacunación $\left({ }^{13}\right)$. Coincidiendo con la información del análisis de 28 artículos científicos que evalúan manifestaciones clínicas de SRC y que incluyeron confirmación por laboratorio (11 prospectivos y 9 retrospectivos) $\left({ }^{14}\right)$, la sordera neurosensorial fue la manifestación clínica de SRC más común, y la más frecuentemente presente en caso de ser defecto único. En nuestro estudio, este último hallazgo no ha sido considerado, por la baja frecuencia de tamizaje para síndrome de TORCH (toxoplasmosis, citomegalovirus, herpes simple y rubéola y la dificultad diagnóstica después del año de edad en pacientes con sordera neurológica, como manifestación única de SRC.

Aproximadamente 50 países en desarrollo han llevado a cabo estudios para conocer la carga bruta de casos de síndrome de rubéola congénita, en 1996. Se encontró 8 casos confirmados de SRC en países del Caribe: Jamaica (6), Barbados (1) y Trinidad y Tobago (1) $\left({ }^{8}\right)$. A estas iniciativas para conocer la carga de enfermedad por el SRC se suma la del Perú, por medio de dos estudios; uno de ellos es el presente estudio, con 2 casos confirmados de SRC, y 5 casos en otra evaluación realizada en 17 establecimientos de salud de tercer y cuarto nivel con atención pediátrica $\left({ }^{15}\right)$.

Los casos encontrados en estos colegios en el Perú (probables y confirmados) representan "la punta del iceberg" de un grupo mayor de niños con SRC, debido a que existen infantes con secuelas de SRC (sordera y ceguera) que, por condiciones estructurales (pobreza, ignorancia, etc.), no acceden a colegios especiales, así como por presentaciones clínicas menos severas; se debe considerar muchas muertes fetales e infantiles que suceden en el hogar dentro de la comunidad y que no son notificadas $\left({ }^{16}\right)$. Según las estimaciones de incidencia anual de casos de SRC en la población rural del Perú de 464 por 100000 nacidos vivos $\left({ }^{7}\right)$ y la información

Tabla 5.- Casos confirmados se síndrome de rubéola congénita.

\begin{tabular}{lll}
\hline \multicolumn{1}{c}{ Caso } & \multicolumn{1}{c}{1} & \multicolumn{1}{c}{2} \\
\hline \multirow{2}{*}{ Establecimiento } & Centro Educativo Nuestra & Centro Educativo Nuestra \\
Edad & Señora Del Carmen & Señora Del Carmen \\
Sexo & 2 años & 6 años \\
Manifestaciones neurológicas & Femenino & Femenino \\
& Sordera neurosensorial & Sordera neurosensorial \\
Manifestaciones cardíacas & Retraso mental & Retraso mental \\
Manifestaciones oftalmológicas & No & No \\
Otros criterios & Antecedente de rash en & Catarata congénita \\
& la madre 1er trimestre & Antecedente de rash en \\
& Peso bajo al nacer & la madre durante 1er trimestre \\
\hline
\end{tabular}


de costos de atención de éstos, costo de vacunas, siguiendo la metodología de un ejercicio de costos de SRC hecho en Trinidad y Tobago $\left({ }^{17}\right)$, el costo aproximado en el Perú para atender a los niños con secuelas de SRC para el año 2002, en el área rural, es 4 veces más que el costo de adquirir la vacuna triple viral $\left({ }^{18}\right)$. En ello no se considera los costos de niños nacidos en el área urbana y de niños nacidos en años anteriores con SRC, que requieren de educación y tratamiento especializados, además de los costos indirectos, tales como el costo emocional de familias cuyo hijo nace con un rango de discapacidades. Éstas incluyen retraso mental, en algunos casos, la disminución de factibilidad de que este individuo contribuya económicamente al desarrollo familiar y del país; además del costo de que este niño muera prematuramente, resultando en un costo de años potenciales de vida perdidos.

Adicionalmente debe tomarse en cuenta los costos directos y emocionales de abortos a causa de SRC, ya sea natural o electivo (e ilegal), y el riesgo de complicaciones, incluso la muerte. La posibilidad de sustituir en el esquema de inmunizaciones la vacuna contra sarampión por la vacuna triple viral (sarampión/parotiditis/ rubéola) e inmunizar a mujeres en edad fértil, podría reducir -significativamente- los casos de rubéola y SRC, e incluso llegar hasta la erradicación, además de prevenir casos de parotiditis y sus complicaciones, contribuyendo así a mejorar la calidad de vida de la población. Debe señalarse que los resultados del presente estudio no son extrapolables a la población general, toda vez que no es un ensayo probabilístico, el tamaño de la muestra es pequeño (no representativo de la población general) y la información se obtuvo de registros médicos. No obstante, la definición de caso utilizada está basada en los criterios para definir casos de SRC, según el CDC $\left.{ }^{(11}\right)$. En el presente estudio, la definición de caso probable coincide con la definición de caso clínicamente confirmado de la Organización Panamericana de la Salud (OPS), utilizada por varios estudios entre ellos uno en Ghana $\left({ }^{16}\right)$.
Puede perderse la oportunidad de reducir o incluso erradicar la rubéola al ligarla al control del sarampión, con un costo marginal relativamente pequeño, si la rubéola congénita permanece sin ser diagnosticada y sin ser reportada.

\section{AGRADECIMIENTOS}

Este estudio se realizó gracias al valioso apoyo del Dr. Percy Minaya León, de la Red Nacional de Epidemiología. Agradecemos especialmente a los responsables de epidemiología de las Direcciones de Salud de Arequipa, Cuzco, Lima Este y Lima Sur, así como las facilidades brindadas por los directivos de colegios especializados C-PAL, San Francisco de Asís, Nuestra Sra. del Pilar en Arequipa, Nuestra Señora del Carmen en Cusco y al servicio de rehabilitación del Hospital Hipólito Unanue.

\section{BIBLIOGRAFÍA}

1) Cooper LZ. The history and medical consequences of rubella. Rev Infect Dis 1985; 7 Suppl 1:S2-10.

2) Plotkin S, Katz M, Cordero J. The erradication of rubella. JAMA 1999; 281(6): 561-2.

3) Rubella and congenital rubella syndrome. Bull WHO 1998; 76 Suppl 2: 156-7.

4) Franklin S, Kelley R. Congenital rubella and intersticial pneumonitis. Clin Ped 2001; 40(2): 101-3.

5) Hinman A, Hersh BA, de Quadros C. Rational use of rubella vaccine for prevention of congenital rubella syndrome in the Americas. Pan Am J Pub Health 1998; 4(3): 156-60.

6) Fuente MESS. Plan de erradicación del sarampión. Oficina General de Epidemiología MINSA, Perú.

7) Manual de Control de Enfermedades Inmuoprevenibles del PAI. MINSA 1995

8) Cutts FT, Robertson SE, Diaz Ortega JL. Control of rubella and congenital rubella syndrome (CRS) in developing countries. Part 1: Burden of disease. Bull WHO 1997; 75(1): $55-6$.

9) Cutts FT, Vinnycky E. Modelling the incidence of congenital rubella syndrome in developing countries. Intern J Epidemiol 1999; 28: 1176-84.

10) Directiva $N^{\circ}$ 001- 99 OGE-OEVEE Misterio de Salud Oficina General de Epidemiologia MINSA. En: Reporte Epidemiológico semanal No 09 (del 28 de febrero al 6 de marzo 1999). Oficina General de Epidemiología. 
11) CDC. Case definitions for infectious conditions under public health surveillance. MMWR 1997; 46[No. RR-10]: 30.

12) Gray RF. Causes of deafness in schools for the deaf in Madras. Intern J Ped Otorr 1989; 18(2): 97-106.

13) Peckham C. Congenital rubella in the United Kingdom before 1970: the prevaccine era. Rev Infect Dis 1985; Suppl 1: s11-6.

14) Reef S, Plotkin S, Cordero J, Katz M, Cooper L, Schwartz B, Zimmerman-Swain L, Danovaro-Holliday M, Wharton M. Preparing for elimination of congenital rubella syndrome (CRS): Summary of a Workshop on CRS Elimination in the United States. Clin Infect Dis 2000; 31: 85-95.

15) Blitchtein D, González P, Gonzales. Estudio aun no publicado.
16) Lawn J, Ref S, Baffoe-Bonnie B, Adadevoh S, Caul EO, Griffin G. Unseen blindness, unheard deafness and unrecorded death and dissability: Congenital rubella in Kumasi, Ghana. Am J Public Health 2000; 90(10): 155561.

17) Lewis MJ, Irons B, Carrasco P, Hersh B, Strassburg M. The Burden of Congenital Rubella Syndrome in the English-speaking Caribbean. XII Meeting of the PAHO Technical Advisory Group on vaccine preventable diseases, Quebec, Canada April 12 - 16, 1999. Division of Vaccines and Immunization, Pan American Health Organization.

18) Documento de trabajo Síndrome de Rubéola Congénita en el Perú: una estimativa de costos. Oficina General de Epidemiologia MINSA. 